\title{
Apparent diffusion coefficient values in borderline breast lesions upgraded and not upgraded at definitive histopathological examination after surgical excision
}

\author{
Corrado Tagliati ${ }^{1 A, B, C, D, E, F}$, Paola Piccinni ${ }^{1 B}$, Paola Ercolani ${ }^{2 D}$, Elisabetta Marconi ${ }^{2 D}$, Barbara Franca Simonetti ${ }^{2 D}$, \\ Gian Marco Giuseppetti ${ }^{3 A, D}$, Andrea Giovagnoni ${ }^{3 D}$ \\ ${ }^{1}$ Università Politecnica delle Marche, Ancona, Italy \\ 2Department of Radiological Sciences, Azienda Ospedaliero Universitaria Ospedali Riuniti, Ancona, Italy \\ ${ }^{3}$ Department of Radiological Sciences, Azienda Ospedaliero Universitaria Ospedali Riuniti, Università Politecnica delle Marche, Ancona, Italy
}

\section{Abstract}

\begin{abstract}
Purpose: The study aims were to evaluate if the apparent diffusion coefficient (ADC) value could distinguish between breast lesions classified as $\mathrm{B} 3$ at core needle biopsy $(\mathrm{CNB})$ that show or do not show atypia or malignancy at definitive histopathological examination (DHE) after surgical excision.
\end{abstract}

\begin{abstract}
Material and methods: From January 2013 to December 2017, 141 patients with a B3 breast lesion underwent magnetic resonance imaging and were included in the study. The $\mathrm{ADC}$ value was assessed drawing a ROI outlining the entire lesion, evaluating the mean $\left(\mathrm{ADC}_{\text {mean }}\right)$ and minimum $\mathrm{ADC}$ values $\left(\mathrm{ADC}_{\text {min }}\right)$.

Results: Both $\mathrm{ADC}_{\text {mean }}$ and $\mathrm{ADC}_{\text {min }}$ values showed a statistically significant difference between $\mathrm{B} 3$ lesions without and with malignancy or, for B3a lesions, atypia at DHE. They both showed a statistically significant difference also between $\mathrm{B} 3 \mathrm{a}$ lesions without or with atypia or malignancy at DHE, but only $\mathrm{ADC}_{\text {min }}$ (not $\mathrm{ADC}_{\text {mean }}$ ) showed statistically significant difference between B3b lesions without or with malignancy at DHE.

Conclusions: The ADC value could help distinguish between B3a lesions without or with atypia/malignancy at DHE after surgical excision and between B3b lesions without or with malignancy at DHE. Therefore, it could be used to help guide the diagnostic-therapeutic pathway of these lesions, particularly of B3a lesions.
\end{abstract}

Key words: borderline breast lesions, B3 lesions, breast MRI, ADC value.

\section{Introduction}

Breast lesions classified as lesions of uncertain malignant potential (B3) are a heterogeneous group of abnormalities with variable risk of associated malignancy. These lesions include atypical ductal hyperplasia (ADH), lobular intraepithelial neoplasia (LIN) 1-2, flat epithelial atypia (FEA), papillary lesions (PL), radial scars/complex sclerosing lesions (RS), and fibroepithelial lesions (FEL) [1]. These lesions can be subclassified according to the ab- sence (B3a) or presence (B3b) of epithelial atypia [2-5]. Image-guided core needle biopsy (CNB) and vacuumassisted biopsy (VAB) are essentials diagnostic procedures, and B3 lesion diagnosis occurs in 4.5-12.8\% of breast specimens [6-8]. However, borderline lesions management is not simple because there is a significant risk of malignancy underestimation $[9,10]$. Surgical excision has been for a long time considered the best treatment option for B3 lesions in order to achieve their definitive characterization. However, recently, the first and second international consensus conference on B3 le-

Correspondence address:

Dr. Corrado Tagliati, Azienda Ospedaliero Universitaria Ospedali Riuniti, Via Conca 71, 60126, Torrette, Ancona, Italy, phone: +390715964078 ,

fax: +39071596 5009, e-mail: corrado.tagliati@gmail.com

Authors' contribution:

A Study design · B Data collection · C Statistical analysis · D Data interpretation · E Manuscript preparation · F Literature search · G Funds collection 
sions recommended the expansion of the field of use of therapeutic VAB $[11,12]$. It is known that the apparent diffusion coefficient (ADC) value could help distinguish between benign, high-risk, and malignant breast lesions [13-17], but no previous studies have evaluated the performance of the ADC value in distinguishing between B3b lesions without and with malignancy at definitive histopathological examination (DHE) after surgical excision, between B3a lesions without and with atypia/malignancy at DHE, and between B3a and B3b lesions.

The study objectives were to evaluate if ADC value could differentiate between B3 and B3a lesions without and with atypia or malignancy at DHE after surgical excision and between B3b lesions without or with malignancy at DHE. Another study aim was to assess breast $\mathrm{ADC}$ value performance in distinguishing between $\mathrm{B} 3 \mathrm{a}$ and B3b lesions.

\section{Material and methods}

\section{Patients}

From January 2013 to December 2017, 3986 CNBs were performed under sonographic guidance, and 384 (9.6\%) B3 lesions were retrospectively collected.

The inclusion criteria were as follows: borderline (B3) lesion diagnosis at CNB; breast magnetic resonance (MR) performed at our hospital between 1 and 2 months after CNB procedure; surgical excision biopsy and pathological examination performed at our hospital within 1 month after MRI and within 3 months after CNB. Exclusion criteria were as follows: concomitant invasive breast cancer or ductal carcinoma in situ (DCIS) in the same breast in which borderline lesion was diagnosed; clinical evidence of haematoma at the CNB site at the time of MRI execution.

According to these criteria, 141 patients (mean age $47.9 \pm 10.0$ years; range 23-71) with 141 B3 lesions were selected by 4 radiologists (T.C., P.P, M.E., and S.B.F.) to be included in this study. Definitive post-surgical histopathological examination showed a maximum lesion diameter between 0.9 and $3.8 \mathrm{~cm}$ (mean $1.4 \pm 0.4 \mathrm{~cm}$ ).

The study was approved by the institutional review board and ethics committee of our institution. Informed consent was obtained from all individual participants included in the study. All procedures performed in studies involving human participants were in accordance with the ethical standards of the institutional and/or national research committee and with the 1964 Helsinki declaration and its later amendments or comparable ethical standards.

\section{Ultrasound-guided core needle biopsy}

All the 141 sonography-guided CNBs were performed using a semi-automatic biopsy gun (Precisa, Hospital Service, Latina, Italy) with a 14-gauge needle, and 4 to
8 (mean 5.5) cores were obtained during each CNB. In all cases local anaesthesia was performed (Lidocaine hydrochloride $20 \mathrm{mg} / \mathrm{ml}$ ). All the lesion showed an important residual component visible at US examination after the procedure (always estimable as about more than 3/4 of the original lesion).

\section{Breast magnetic resonance imaging protocol}

Breast MRI was performed with a $1.5 \mathrm{~T}$ system (Achieva, Philips Medical Systems, Andover, MA) and a dedicated breast coil (SENSE Breast 7), using a standard protocol including the following sequences, each one with a field of view (FOV) of $340 \times 340 \mathrm{~mm}$ :

- axial short tau inversion recovery (STIR) sequence with a TR/TE of 7758/70; flip angle $90^{\circ}$; matrix size of $272 \times 222$; slice thickness of $3 \mathrm{~mm}$; slice spacing of $3 \mathrm{~mm}$; acquisition time of 4 minutes 39 seconds;

- axial diffusion-weighted imaging (DWI) with a single-shot echo-planar imaging (EPI); $b$ value of 0 and $800 \mathrm{~s} / \mathrm{mm}^{2}$; TR/TE of $13,811 / 86$; matrix size of $228 \times 226$; $2 \mathrm{NEX}$; slice thickness of $3 \mathrm{~mm}$; slice spacing of $3 \mathrm{~mm}$ and an acquisition time of 3 minutes 27 seconds;

- axial T1-weighted 3D fat-suppressed (T1fs), spoiled gradient-echo sequence (THRIVE) with TR/TE of 6.9/3.4; flip angle $12^{\circ}$; matrix size of $320 \times 318$; isotropic voxel of $1 \mathrm{~mm}$; acquisition time of 1 minute 17 seconds for each phase and a total acquisition time of 7 minutes and 53 second; 1 phase before and 5 phases after gadobenate dimeglumine (Dotarem; Guerbet BP 57400, F-95943 RoissyCdGCedex, France) bolus injection $(0.2 \mathrm{ml} / \mathrm{kg}$, $0.1 \mathrm{mmol} / \mathrm{kg}$ injected at $2.0 \mathrm{ml} / \mathrm{s}$ ) followed by flushing with $20 \mathrm{ml}$ of saline at $2.0 \mathrm{ml} / \mathrm{s}$ using an electronic power injector (Spectris MR Injector, Medrad).

Subtraction, multiplanar reconstruction (MPR) and maximum intensity projection (MIP) images, and ADC maps were also obtained.

\section{Report evaluation}

Two radiologists with at least 10 years' experience in breast MRI (G.G.M. and E.P.), who were blinded to the lesion definitive histopathological examination reports, assessed the MR images in consensus.

As regards quantitative ADC values, once the lesion was identified on DWI, a region of interest (ROI) was manually placed in each slice in which the lesion was detectable on the $b=800 \mathrm{~s} / \mathrm{mm}^{2}$ DWI to encompass as much of the abnormality as possible, while staying within its border and taking care to avoid haemorrhagic and cystic areas [18]. Mean $\mathrm{ADC}$ value $\left(\mathrm{ADC}_{\text {mean }}\right)$, which corresponds to the area-weighted average of the ADC values found within each lesion slice, and minimum ADC value $\left(\mathrm{ADC}_{\text {min }}\right)$, which corresponds to the lower $\mathrm{ADC}$ value found among the different slices, were used in the analysis. 


\section{Statistical method}

Definitive histopathological examinations were used as a gold standard in order to evaluate ADC value differences among B3 lesions. B3 lesions without epithelial atypia at $\mathrm{CNB}$ were considered $\mathrm{B} 3 \mathrm{a}$ (PL, FEL, and RS without atypia), and the ones with epithelial atypia were considered B3b (ADH, LIN, FEA or PL, FEL, and RS with atypia at CNB). At DHE after surgical excision these lesions were further categorized in B3a without or with atypia or malignancy, and B3b without or with malignancy.

$\mathrm{ADC}$ values differences between groups were evaluated using $t$-test. The ADC threshold was chosen by the area under the ROC curve (AUC) and Youden index. AUCs were compared through the $z$-test. Qualitative variables were evaluated using the non-parametric Mann-Whitney $U$ test. The statistical significance level was set at $p<0.05$. All statistical analyses were performed using MedCalc Software v. 15.8 (Ostend, BEL).

\section{Results}

\section{Histopathological findings}

$\mathrm{CNB}$ diagnosis and specific CNB underestimation rates are reported in Table 1 . The overall CNB underestimation rate was $25.5 \%$, and the malignancy underestimation rate was $15.6 \%$. DHE showed 16 DCISs, 4 IDCs, and 2 ILCs. 10/34 ADHs were reported as malignant (7 DCISs, 3 IDCs); 2/7 FEAs showed DCIS at DHE; 5/21 LINs were reported as malignant at DHE (2 DCISs, 2 ILCs, 1 IDCs); 13/63 PLs were reported as atypical at DHE, and 4/63 showed DCIS; 1/13 FELs showed DCIS, and 1/13 LINs.

\section{Magnetic resonance imaging findings}

All the 141 B3 lesions were visible on DWI and ADC map. Therefore, all the 141 lesions were included in the ADC value analysis. $\mathrm{ADC}_{\text {mean }}$ and $\mathrm{ADC}_{\text {min }}$ values of $\mathrm{B} 3$ lesions without (WO) and with (W) upgrade at DHE are reported in Table 2 (Figures 1-3).

\section{Statistical analysis}

$\mathrm{ADC}_{\text {mean }}$ and $\mathrm{ADC}_{\text {min }}$ values differences among $\mathrm{B} 3$ lesions are reported in Table 3.

$\mathrm{ADC}_{\text {mean }}$ and $\mathrm{ADC}_{\text {min }}$ AUC comparisons are shown in Figure 4.

\section{Discussion}

DWI, quantified by ADC value, is an MRI technique that is used to evaluate the 3-dimensional mobility of water in vivo and enables indirect assessment of tissue microstructure. Advances in MR technology including echo-planar imaging, multichannel coils, and parallel imaging allowed the extension of the use of DWI in breast imaging, where it was first applied in 1997 by Englander et al. [18, 19].

In this study, $\mathrm{ADC}$ values were statistically different between B3 and B3a lesions without or with atypia or malignancy at DHE after surgical excision, between B3b lesions without or with malignancy at DHE, and between $\mathrm{B} 3 \mathrm{a}$ and $\mathrm{B} 3 \mathrm{~b}$ lesions. The present study showed a better performance of the $\mathrm{ADC}_{\text {min }}$ value than $\mathrm{ADC}_{\text {mean }}$ in predicting B3b lesions with malignancy at DHE or B3a lesions with atypia/malignancy at DHE.

The higher cellularity and the architectural changes of B3 lesions with associated atypia or malignancy could be the cause of the lower mean ADC values of these lesions, because in these conditions the motion of water molecules is more restricted [20-22].

Diffusion restriction and low ADC values have been correlated with the presence of malignant lesions on non-

Table 1. Borderline lesions underestimation rate at definitive histopathological examination

\begin{tabular}{|l|c|c|}
\hline $\begin{array}{l}\text { CNB } \\
\text { diagnosis }\end{array}$ & $\begin{array}{c}\text { Number of } \\
\text { lesions at CNB }\end{array}$ & $\begin{array}{c}\text { Underestimation rate at DHE: } \\
\text { atypia* or malignancy** }\end{array}$ \\
\hline ADH & 34 & $10(29.4 \%): 10^{* *}(29.4 \%)$ \\
\hline LIN 1-2 & 21 & $5(23.8 \%): 5^{* *}(23.8 \%)$ \\
\hline FEA & 7 & $2(28.6 \%): 2^{* *}(28.6 \%)$ \\
\hline PL & 63 & $17(27.0 \%): 13^{*}(20.6 \%), 4^{* *}(6.4 \%)$ \\
\hline RS & 3 & $0(0 \%)$ \\
\hline FEL & 13 & $2(15.4 \%): 1^{*}(7.7 \%), 1^{* *}(7.7 \%)$ \\
\hline Total & 141 & $36(25.5 \%): 14^{*}(9.9 \%), 22^{* *}(15.6 \%)$ \\
\hline
\end{tabular}

CNB - core needle biopsy, ADH - atypical ductal hyperplasia, LIN 1-2 - lobular intraephitelial neoplasia 1 or 2, FEA - flat epithelial atypia, PL - papillary lesion, RS - radial scar, FEL - fibroepithelial lesion

Table 2. $B 3$ lesions $A D C_{\text {mean }}$ and $A D C_{\text {min }}$ values

\begin{tabular}{|l|l|l|}
\hline B3 lesions (n.) & $\begin{array}{l}A D C_{\text {man }} \pm \text { SD } \\
\times 10^{-3} \mathrm{~mm}^{2} / \mathrm{s}\end{array}$ & $\begin{array}{l}\mathrm{ADC}_{\min } \pm \mathrm{SD} \\
\times 10^{-3} \mathrm{~mm}^{2} / \mathrm{s}\end{array}$ \\
\hline B3a+b (141) & $1.417 \pm 0.130$ & $1.002 \pm 0.170$ \\
\hline B3a (79) & $1.458 \pm 0.145$ & $1.081 \pm 0.177$ \\
\hline B3b (62) & $1.365 \pm 0.085$ & $0.902 \pm 0.090$ \\
\hline B3a+bW0 (105) & $1.433 \pm 0.133$ & $1.052 \pm 0.163$ \\
\hline B3a+bW (36) & $1.370 \pm 0.111$ & $0.860 \pm 0.091$ \\
\hline B3aW0 (60) & $1.490 \pm 0.131$ & $1.152 \pm 0.126$ \\
\hline B3aW (19) & $1.354 \pm 0.139$ & $0.854 \pm 0.113$ \\
\hline B3bW0 (45) & $1.357 \pm 0.089$ & $0.915 \pm 0.096$ \\
\hline B3bW (17) & $1.387 \pm 0.069$ & $0.866 \pm 0.061$ \\
\hline
\end{tabular}

$A D C$ - apparent diffusion coefficient, $S D$ - standard deviation, $B 3 a+b W 0$ - B3 lesions without upgrade at definitive histopathological examination (DHE), B3a+bW - B3 lesions with upgrade at DHE (upgrade to malignancy for B3b or, to malignancy or atypia for B3a lesions), B3aWO - B3a lesions without atypia or malignancy at DHE, B3aW - B3a lesions with atypia or malignancy at DHE, B3bWO - B3b lesions without malignancy at DHE, B3bW - B3b lesions with malignancy at DHE 


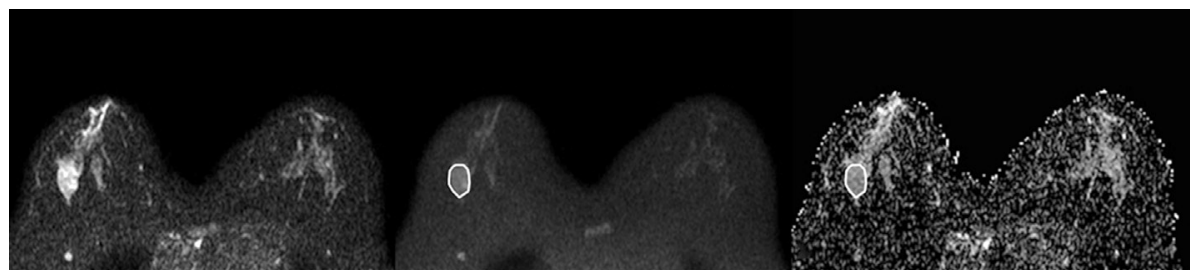

Figure 1. A 42-year-old woman with a 3.2-cm lesion in her right breast. The lesion appeared hyperintense on DWls obtained at $b=0(\mathrm{~A})$ and $b=800(\mathrm{~B})$, and hypointense on $\mathrm{ADC}$ map $\left(\mathrm{ADC}_{\text {mean }} 1.251 \times 10^{-3} \mathrm{~mm}^{2} / \mathrm{s}, A D C_{\text {mi }}\right.$ $0.726 \times 10^{-3} \mathrm{~mm}^{2} / \mathrm{s}$ ). It was diagnosed as fibroepithelial lesion at core needle biopsy and upgraded to ductal carcinoma in situ at definitive histopathological examination after surgical excision

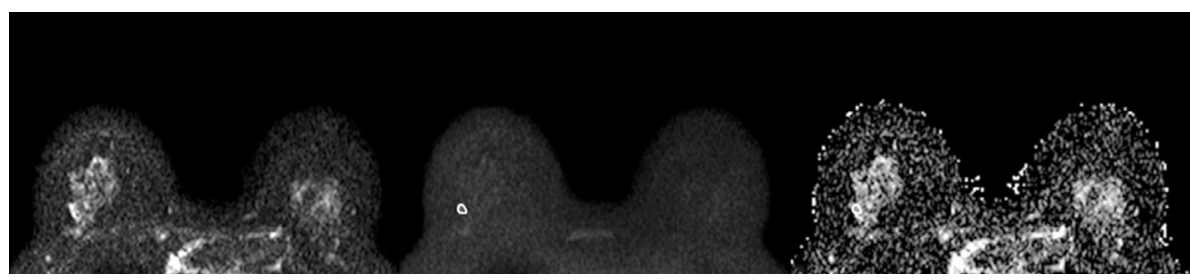

Figure 2. A 43-year-old woman with a 1.0-cm lesion in her right breast. The lesion appeared slightly hyperintense on DWls obtained at $b=0(\mathrm{~A})$ and $b=800(\mathrm{~B})$, and hypointense on $\mathrm{ADC}$ map $\left(\mathrm{ADC}\right.$ mean $1.413 \times 10^{-3} \mathrm{~mm}^{2} / \mathrm{s}$, $A D C_{\min } 1.065 \times 10^{-3} \mathrm{~mm}^{2} / \mathrm{s}$ ). It was diagnosed as lobular intra-ephitelial neoplasia (LIN) 1 at core needle biopsy and was confirmed to be LIN1 at definitive histopathological examination after surgical excision

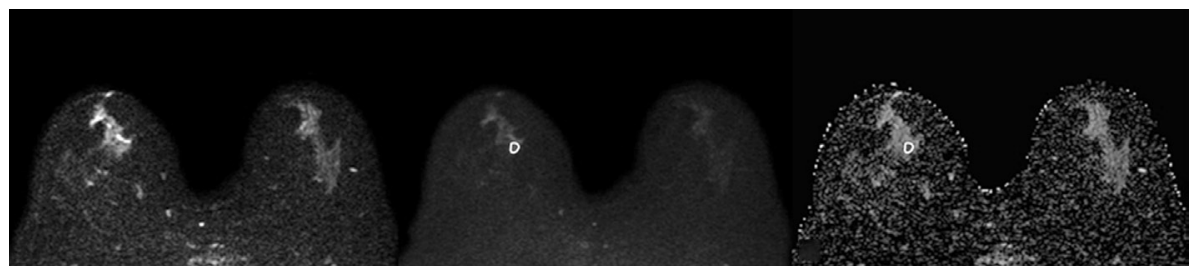

Figure 3. A 62-year-old woman with a 1.1-cm lesion in her right breast. The lesion appeared slightly hyperintense on DWls obtained at $b=0(A)$ and $b=800(B)$, and hypointense on $A D C$ map $\left(A D C_{\text {mean }} 1.081 \times 10^{-3} \mathrm{~mm}^{2} / \mathrm{s}\right.$, $\left.A D C_{\min } 0.722 \times 10^{-3} \mathrm{~mm}^{2} / \mathrm{s}\right)$. It was diagnosed as atypical ductal hyperplasia at vacuum-assisted biopsy and upgraded to IDC at definitive histopathological examination after surgical excision

Table 3. $A D C_{\text {mean }}$ and $A D C_{\text {min }}$ values differences among $B 3$ lesions

\begin{tabular}{|c|c|c|c|c|c|c|}
\hline$A D C$ & B3 lesions & $p$ & $\begin{array}{l}\text { Threshold } \\
\times 10^{-3} \mathrm{~mm}^{2} / \mathrm{s}\end{array}$ & Sensitivity & Specificity & AUC \\
\hline \multirow[t]{4}{*}{$A D C_{\text {mean }}$} & B3a vs. B3b & $<0.0001$ & 1.401 & 64.5 & 65.8 & 0.716 \\
\hline & $B 3 a+b W 0$ vs. B3a+bW & 0.0074 & 1.398 & 61.1 & 62.9 & 0.636 \\
\hline & B3aW0 vs. B3aW & $<0.0001$ & 1.418 & 68.4 & 73.3 & 0.757 \\
\hline & B3bW0 vs. B3bW & 0.2814 & 1.381 & 58.8 & 48.9 & 0.590 \\
\hline \multirow[t]{4}{*}{$A D C_{\min }$} & B3a vs. B3b & $<0.0001$ & 0.954 & 80.7 & 76.0 & 0.795 \\
\hline & $B 3 a+b W 0$ vs. B3a+bW & $<0.0001$ & 0.903 & 77.8 & 77.1 & 0.849 \\
\hline & B3aW0 vs. B3aW & $<0.0001$ & 0.923 & 89.5 & 96.7 & 0.956 \\
\hline & B3bW0 vs. B3bW & 0.0271 & 0.869 & 58.8 & 75.6 & 0.663 \\
\hline
\end{tabular}

$A D C$ - apparent diffusion coefficient, AUC - area under the ROC curve, B3a+bWO - B3 lesions without upgrade at definitive histopathological examination (DHE), B3a+bW - B3 lesions with upgrade at DHE (upgrade to malignancy for B3b or, to malignancy or atypia for B3a lesions), B3aWO - B3a lesions without atypia or malignancy at DHE, B3aW - B3a lesions with atypia or malignancy at DHE, B3bWO - B3b lesions without malignancy at DHE, B3bW - B3b lesions with malignancy at DHE

selected subjects [23-32], and previous studies showed that ADC values of normal breast tissue, benign lesions, and malignant lesions are related to the technical parameters of acquisition of MRI examination [33,34]. A previous study demonstrated that the mean $\mathrm{ADC}$ is significantly different between benign lesions and high-risk lesions [35].

In this study the patients underwent an MRI examination with a maximum DWI $b$ value of $800 \mathrm{~s} / \mathrm{mm}^{2}$. This 

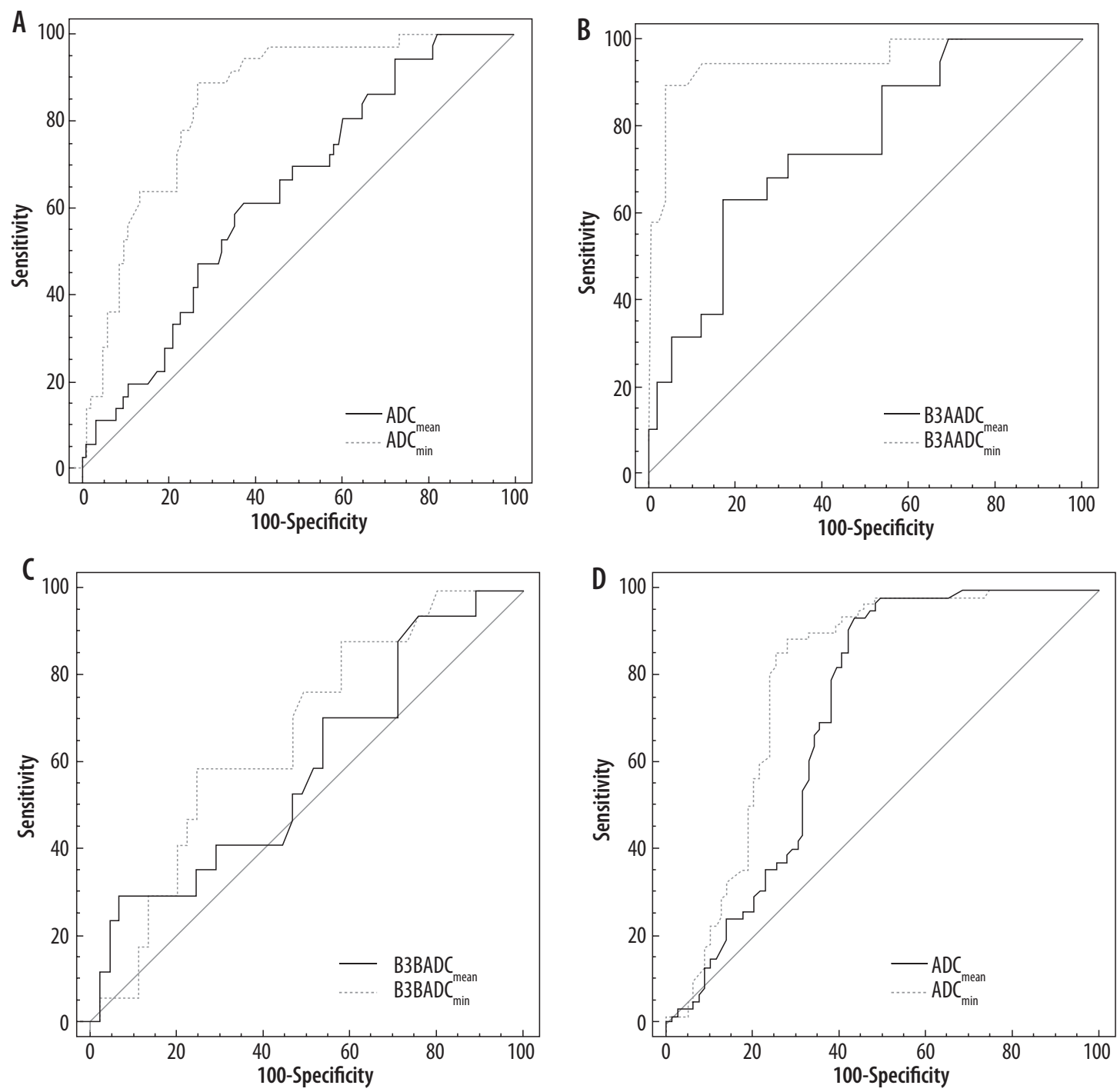

Figure 4. $A U C A D C_{\text {mean }}$ and $A D C_{\text {min }}$ comparison between: $A$ ) $B 3$ lesions upgraded and not upgraded (to malignancy for $B 3 b$, and to malignancy or atypia for B3a at definitive histopathological examination showed statistically significant difference ( $p<0.0001, z$ statistic 4.289$)$; B) B3a lesions upgraded and not upgraded to malignancy or atypia at definitive histopathological examination showed statistically significant difference $(p=0.0005, z$ statistic 3.508$)$; C) B3b lesions upgraded and not upgraded to malignancy at definitive histopathological examination did not showed statistically significant difference $(p=0.5120$, z statistic 0.653$)$; D) B3a and B3b lesions did not showed statistically significant difference ( $p=0.0692, z$ statistic 1.817$)$

value is commonly used in breast imaging [27] and was used in a previous study in this field [36].

To our knowledge, this is the first study that evaluated and found $\mathrm{ADC}$ value differences between $\mathrm{B} 3 \mathrm{~b}$ lesions without and with malignancy at DHE after surgical excision, or between B3a lesions without and with atypia /malignancy at DHE, regardless of BI-RADS category. Moreover, this study found $\mathrm{ADC}$ value differences between $\mathrm{B} 3 \mathrm{a}$ and $\mathrm{B} 3 \mathrm{~b}$ lesions.

Only 1 previous study assessed and found a significant correlation between B3 lesions upgraded to malignancy at surgery and $\mathrm{ADC}$ value, but the ADC value was evaluated in 23 breast lesions classified as MRI BI-RADS categories 4 and 5 only [36].

A previous study reported a better performance of $\mathrm{ADC}_{\text {min }}$ value than $\mathrm{ADC}_{\text {mean }}$ in distinguish between benign and malignant lesions, and our study confirmed a statistically significant difference between $\mathrm{ADC}_{\text {min }}$ and
$\mathrm{ADC}_{\text {mean }}$ performance, although in another setting, because it was reached in 2 out of 4 cases [37].

Regarding the possible clinical implications of our findings, we think that the ADC value, especially the $\mathrm{ADC}_{\text {min }}$ value, could be used to help guide the diagnostictherapeutic pathway of these lesions. For example, B3 lesions with an ADC value under the thresholds reported in this study could be worthy of surgical excision or more frequent follow-up after VAB excision when the same MR scan and the same sequence settings are used. This could be particularly useful in patients who would otherwise not be referred for surgical excision or strict follow-up based on CNB results. In particular, our results show a possible clinical use of $\mathrm{ADC}_{\min }$ in $\mathrm{B} 3 \mathrm{a}$ lesions, in order to help discriminate those lesions that show atypical or malignant foci at DHE. Confirmation of such findings from future prospective studies is warranted. 
The present study has some limitations. First of all, it is a single-centre retrospective analysis, with a limited number of patients. Furthermore, ADC value inter-observer variability, MRI BIRADS descriptors and assessment categories were not assessed. Moreover, we are aware of the fact that performing MRI after CNB could be a shortcoming. However, this could be considered more useful in a clinical setting, because in daily practice MRI examinations are more frequently performed after this procedures.

\section{Conclusions}

The ADC value could help distinguish between B3 lesions without and with atypia or malignancy at definitive histopathological examination, and it may be a useful tool to help guide the management of $\mathrm{B} 3$ lesions.

\section{Conflict of interest}

The authors report no conflict of interest.

\section{References}

1. Lakhani SR, Ellis IO, Schnitt SJ, et al. WHO classification of tumours of the breast. $4^{\text {th }}$ edition. Lyon: International Agency for Research on Cancer; 2012.

2. Mayer S, Kayser G, Rücker G, et al. Absence of epithelial atypia in $\mathrm{B} 3$-lesions of the breast is associated with decreased risk for malignancy. Breast 2017; 31: 144-149.

3. de Beça FF, Rasteiro C, Correia A, et al. Improved malignancy prediction by B3 breast lesions subclassification. Ann Diagn Pathol 2013; 17: 434-436.

4. Rakha EA, Lee AH, Jenkins JA, et al. Characterization and outcome of breast needle core biopsy diagnoses of lesions of uncertain malignant potential (B3) in abnormalities detected by mammographic screening. Int J Cancer 2011; 129: 1417-1424.

5. Ibrahim AE, Bateman AC, Theaker JM, et al. The role and histological classification of needle core biopsy in comparison with fine needle aspiration cytology in the preoperative assessment of impalpable breast lesions. J Clin Pathol 2001; 54: 121-125.

6. Rakha EA, Ho BC, Naik V, et al. Outcome of breast lesions diagnosed as lesion of uncertain malignant potential (B3) or suspicious of malignancy (B4) on needle core biopsy, including detailed review of epithelial atypia. Histopathology 2011; 58: 626-632.

7. Andreu FJ, Sáez A, Sentís M, et al. Breast core biopsy reporting categories - an internal validation in a series of 3054 consecutive lesions. Breast 2007; 16: 94-101.

8. Pagni F, Bosisio FM, Salvioni D, et al. Application of the British National Health Service Breast Cancer Screening Programme classification in 226 breast core needle biopsies: correlation with resected specimens. Ann Diagn Pathol 2012; 16: 112-118.

9. Perry N, Broeders M, de Wolf C, et al. European guidelines for quality assurance in breast cancer screening and diagnosis. Fourth edition - summary document. Ann Oncol 2008; 19: 614-622.

10. Sohn YM, Seo M. Breast lesions diagnosed by ultrasound-guided core needle biopsy: can shearwave elastography predict histologic upgrade after surgery or vaccuum assisted excision? Clin Imaging 2018; 49: 150-155.

11. Rageth CJ, O’Flynn EA, Comstock C, et al. First International Consensus Conference on lesions of uncertain malignant potential in the breast (B3 lesions). Breast Cancer Res Treat 2016; 159: 203-213.

12. Rageth CJ, O’Flynn EAM, Pinker K, et al. Second International Consensus Conference on lesions of uncertain malignant potential in the breast (B3 lesions). Breast Cancer Res Treat 2019; 174: 279-296.
13. Jansen SA, Fan X, Karczmar GS, et al. Differentiation between benign and malignant breast lesions detected by bilateral dynamic contrast-enhanced MRI: a sensitivity and specificity study. Magn Reson Med 2008; 59: 747-754.

14. Yin J, Yang J, Jiang Z. Discrimination between malignant and benign mass-like lesions from breast dynamic contrast enhanced MRI: semi-automatic vs. manual analysis of the signal time-intensity curves. J Cancer 2018; 9: 834-840.

15. Kim SG, Freed M, Leite APK, et al. Separation of benign and malignant breast lesions using dynamic contrast enhanced MRI in a biopsy cohort. J Magn Reson Imaging 2017; 45: 1385-1393.

16. Nogueira L, Brandão S, Matos E, et al. Diffusion-weighted imaging: determination of the best pair of $b$-values to discriminate breast lesions. Br J Radiol 2014; 87: 20130807.

17. Parsian S, Rahbar H, Allison KH, et al. Nonmalignant breast lesions: ADCs of benign and high-risk subtypes assessed as false-positive at dynamic enhanced MR imaging. Radiology 2012; 265: 696-706.

18. Partridge SC, McDonald ES. Diffusion weighted magnetic resonance imaging of the breast: protocol optimization, interpretation, and clinical applications. Magn Reson Imaging Clin N Am 2013; 21: 601-624.

19. Englander SA, Uluğ AM, Brem R, et al. Diffusion imaging of human breast. NMR Biomed 1997; 10: 348-352.

20. Woodhams R, Ramadan S, Stanwell P, et al. Diffusion-weighted imaging of the breast: principles and clinical applications. Radiographics 2011; 31: 1059-1084.

21. Ramírez-Galván YA, Cardona-Huerta S, Ibarra-Fombona E, ElizondoRiojas G. Apparent diffusion coefficient (ADC) value to evaluate BI-RADS 4 breast lesions: correlation with pathological findings. Clin Imaging 2015; 39: 51-55.

22. Kuroki Y, Nasu K. Advances in breast MRI: diffusion-weighted imaging of the breast. Breast Cancer 2008; 15: 212-217.

23. Peters NH, Vincken KL, van den Bosch MA, et al. Quantitative diffusion weighted imaging for differentiation of benign and malignant breast lesions: the influence of the choice of b-values. J Magn Reson Imaging 2010; 31: 1100-1105.

24. Woodhams R, Matsunaga K, Kan S, et al. ADC mapping of benign and malignant breast tumors. Magn Reson Med Sci 2005; 4: 35-42.

25. Deike-Hofmann K, Kuder T, König F, et al. Diffusion-weighted breast imaging. Radiologe 2018; 58 (Suppl 1): 14-19.

26. Yılmaz E, Sarı O, Yılmaz A, et al. Diffusion-weighted imaging for the discrimination of benign and malignant breast masses; utility of ADC and relative ADC. J Belg Soc Radiol 2018; 102: 24. 
27. Partridge SC, Amornsiripanitch N. DWI in the assessment of breast lesions. Top Magn Reson Imaging 2017; 26: 201-209.

28. Partridge SC, Nissan N, Rahbar H, et al. Diffusion-weighted breast MRI: clinical applications and emerging techniques. J Magn Reson Imaging 2017; 45: 337-355.

29. Spick C, Bickel H, Pinker K, et al. Diffusion-weighted MRI of breast lesions: a prospective clinical investigation of the quantitative imaging biomarker characteristics of reproducibility, repeatability, and diagnostic accuracy. NMR Biomed 2016; 29: 1445-1453.

30. Roknsharifi S, Fishman MDC, Agarwal MD, et al. The role of diffusion weighted imaging as supplement to dynamic contrast enhanced breast MRI: can it help predict malignancy, histologic grade and recurrence? Acad Radiol 2019; 26: 923-929.

31. Jin G, An N, Jacobs MA, Li K. The role of parallel diffusion-weighted imaging and apparent diffusion coefficient (ADC) map values for evaluating breast lesions: preliminary results. Acad Radiol 2010; 17: 456-463.

32. Kuroki Y, Nasu K. Advances in breast MRI: diffusion-weighted imaging of the breast. Breast Cancer 2008; 15: 212-217.
33. Shi RY, Yao QY, Wu LM, Xu JR. Breast lesions: diagnosis using diffusion weighted imaging at $1.5 \mathrm{~T}$ and $3.0 \mathrm{~T}$ - systematic review and meta-analysis. Clin Breast Cancer 2018; 18: e305-320.

34. Dong H, Li Y, Li H, et al. Study of the reduced field-of-view diffusion-weighted imaging of the breast. Clin Breast Cancer 2014; 14: 265-271.

35. Parsian S, Rahbar H, Allison KH, et al. Nonmalignant breast lesions: ADCs of benign and high-risk subtypes assessed as false-positive at dynamic enhanced MR imaging. Radiology 2012; 265: 696-706.

36. Cheeney S, Rahbar H, Dontchos BN, et al. Apparent diffusion coefficient values may help predict which MRI-detected high-risk breastlesions will upgrade at surgical excision. J Magn Reson Imaging 2017; 46: 1028-1036.

37. Zhang W, Jin GQ, Liu JJ, et al. Diagnostic performance of ADCs in different ROIs for breast lesions. Int J Clin Exp Med 2015; 8: 12096 12104. 